ARTICLE

https://doi.org/10.1038/s41467-019-11825-2

\title{
Mechanical-force-induced non-local collective ferroelastic switching in epitaxial lead-titanate thin films
}

\author{
Xiaoyan Lu (10 1, Zuhuang Chen ${ }^{2}$, Ye Cao ${ }^{3}$, Yunlong Tang ${ }^{4}$, Ruijuan $\mathrm{Xu}^{4}$, Sahar Saremi (i) ${ }^{4}$, Zhan Zhang ${ }^{5}$, \\ Lu You (1) ${ }^{6}$, Yongqi Dong ${ }^{5}$, Sujit Das ${ }^{4}$, Hangbo Zhang ${ }^{1}$, Limei Zheng ${ }^{7}$, Huaping Wu ${ }^{8}$, Weiming Lv ${ }^{7}$, \\ Guoqiang Xie ${ }^{2}$, Xingjun Liu ${ }^{2}$, Jiangyu Li ${ }^{9}$, Lang Chen (10 ${ }^{10}$, Long-Qing Chen ${ }^{11}$, Wenwu Cao (D) ${ }^{7,12} \&$ \\ Lane W. Martin (1) 4,13
}

Ferroelastic switching in ferroelectric/multiferroic oxides plays a crucial role in determining their dielectric, piezoelectric, and magnetoelectric properties. In thin films of these materials, however, substrate clamping is generally thought to limit the electric-field- or mechanicalforce-driven responses to the local scale. Here, we report mechanical-force-induced largearea, non-local, collective ferroelastic domain switching in $\mathrm{PbTiO}_{3}$ epitaxial thin films by tuning the misfit-strain to be near a phase boundary wherein $c / a$ and $a_{1} / a_{2}$ nanodomains coexist. Phenomenological models suggest that the collective, $c-a-c-a$ ferroelastic switching arises from the small potential barrier between the degenerate domain structures, and the large anisotropy of $a$ and $c$ domains, which collectively generates much larger response and large-area domain propagation. Large-area, non-local response under small stimuli, unlike traditional local response to external field, provides an opportunity of unique response to local stimuli, which has potential for use in high-sensitivity pressure sensors and switches.

\footnotetext{
${ }^{1}$ School of Civil Engineering, Harbin Institute of Technology, Harbin 150001, China. ${ }^{2}$ School of Materials Science and Engineering, Harbin Institute of Technology, Shenzhen 518055, China. ${ }^{3}$ Departments of Materials Science and Engineering, University of Texas at Arlington, Arlington, TX 76019 , USA. ${ }^{4}$ Department of Materials Science and Engineering, University of California, Berkeley, CA 94720, USA. ${ }^{5}$ X-ray Science Division, Advanced Photon Source, Argonne National Laboratory, Argonne, IL 60439, USA. ${ }^{6}$ School of Materials Science and Engineering, Nanyang Technological University, Singapore 639798, Singapore. ${ }^{7}$ Condensed Matter Science and Technology Institute, Harbin Institute of Technology, Harbin 150080, China. ${ }^{8}$ Key Laboratory of E\&M (Zhejiang University of Technology), Ministry of Education \& Zhejiang Province, Hangzhou 310014, China. ${ }^{9}$ Department of Mechanical Engineering, University of Washington, Seattle, WA 98195-2600, USA. ${ }^{10}$ Department of Physics, Southern University of Science and Technology, Shenzhen 518055, China.

${ }^{11}$ Department of Materials Science and Engineering, The Pennsylvania State University, University Park, PA 16802, USA. ${ }^{12}$ Department of Mathematics and Materials Research Institute, The Pennsylvania State University, University Park, PA 16802, USA. ${ }^{13}$ Materials Sciences Division, Lawrence Berkeley National Laboratory, Berkeley, CA 94720, USA. Correspondence and requests for materials should be addressed to X.L. (email: luxy@hit.edu.cn) or to Z.C. (email: zuhuang@hit.edu.cn) or to L.W.M. (email: Iwmartin@berkeley.edu)
} 
$\mathrm{D}$ omain structure and its switching behavior are crucial to material properties, including dielectric and piezoelectric response in ferroelectrics and magnetoelectric coupling in multiferroics ${ }^{1-3}$. In particular, for ferroelectrics, ferroelastic switching (i.e., non- $180^{\circ}$ switching events) can give rise to large dielectric and electromechanical responses due to strong lattice strain-polarization coupling ${ }^{4-7}$. Furthermore, large-area ferroelastic switching under small stimuli can also be vital for magnetoelectric coupling in multiferroics, which are being considered for low-power electric field-controlled spintronics ${ }^{8-10}$. Large-area ferroelastic switching in ferroelectrics, however, has typically only been observed in bulk materials ${ }^{11-13}$. In fact, it is generally thought that ferroelastic switching is quenched in ferroelectric epitaxial thin films due to substrate constraints ${ }^{14-16}$. In order to reduce substrate clamping and facilitate ferroelastic domain switching, several approaches have been explored ${ }^{17-24}$. For example, by fabricating thin films into micro- or nanoscale islands with lateral dimensions on the order of the thickness of the film, researchers have released lateral constraint from the substrate, thereby enabling larger fractions of ferroelastic switching ${ }^{4,9}$. Such approaches require lithography and/or milling/ etching, both of which are time-consuming and challenging. Furthermore, the lateral sizes of the features must be very small, limiting the ability to produce ferroelastic switching across large areas. Therefore, despite considerable efforts, it remains a challenge to achieve large-area ferroelastic switching in ferroelectric epitaxial thin films.

It is also known that long-range interactions (i.e., dipole-dipole electrostatic and elastic interactions) in ferroelectrics could induce collective behavior during domain switching ${ }^{25-30}$. For instance, phenomenological approaches have revealed that the electrostatic interaction between switched nuclei in ferroelectric thin films with $180^{\circ}$ stripe domains can be long range and induce collective nucleation/switching, thereby effectively reducing the switching barrier ${ }^{27}$. Moreover, the domain-switching process in ferroelectric ceramics is thought to be a highly correlated collective process such that the switching process in one grain affects that in the neighboring grains because of inter-grain elastic interactions ${ }^{28}$. Such collective behavior and any resulting large responses would be more evident in ferroelectrics perched near a phase boundary wherein two phases are nearly energetically degenerate and can be interconverted by small external stimuli ${ }^{28-31}$. While most observations of collective switching behavior have been observed in bulk or polycrystalline thin films of ferroelectrics $^{28-30,32}$, work in epitaxial thin films has shown that ferroelastic switching, with enhanced piezoelectric response, can be obtained in thin films of tetragonal ferroelectrics such as $\mathrm{PbZr}_{0.2} \mathrm{Ti}_{0.8} \mathrm{O}_{3}$ by fabrication of micro- or nanoscale islands ${ }^{3,33}$. More recently, ferroelastic switching was also observed in tetragonal ferroelectric thin films when they were grown on the right lattice-(mis)matched substrates ${ }^{18,19}$. At the same time, the use of scanning-probe excitation has also opened the door to induce and control ferroelastic switching via a combination of applied voltages and tip motion ${ }^{4,34,35}$. Despite these advances, however, previous studies have illustrated only local ferroelastic domainswitching behaviors (i.e., directly under or very close to the poling region). It is generally well accepted that such effects will be highly localized as the elastic clamping of the substrate limits the ability to create large-scale ferroelastic changes in the domain structure.

Recent studies have identified epitaxial strain approaches to create multiple nanoscale-domain structures co-existing in $\mathrm{PbTiO}_{3}$ thin films ${ }^{36}$ which provides an intriguing system to explore in this regard. More specifically, 40-nm-thick, (001)oriented films of $\mathrm{PbTiO}_{3}$ grown on $\mathrm{DyScO}_{3}(110)_{O}$ (where the $O$ denotes orthorhombic indices) experience a compressive strain that drives the formation of traditional $c / a$ domain structures, while films grown on $\mathrm{NdScO}_{3}(110)_{O}$ substrates with large tensile strain exhibit $a_{1} / a_{2}$ domain structures, and films grown on $\mathrm{SmScO}_{3}(110)_{O}$ substrates with a strain state between that of $\mathrm{DyScO}_{3}$ and $\mathrm{NdScO}_{3}$ exhibit a coexistence of both $c / a$ and $a_{1} / a_{2}$ domain variant ${ }^{36}$. In effect, epitaxial strain can be used to place this material on the brink of a transition between domainstructure variants and is an ideal route by which to explore the potential for large responses and collective effects ${ }^{37,38}$.

Here, we focus on $\mathrm{PbTiO}_{3}$ epitaxial heterostructures with coexisting $c / a$ and $a_{1} / a_{2}$ nanodomains by tuning the misfit strain to be near a phase boundary. Electric field-poling studies via scanning-probe microscopy reveal that reversible and localized ferroelastic switching can be achieved by fine control of the outof-plane poling voltage. Local mechanical force induced by the tip of an atomic force microscope, on the other hand, can drive largearea, non-local ferroelastic switching-much larger than the contact area. Using Landau phenomenological theory including phase-field simulation $^{39}$ and polydomain theory ${ }^{40}$, further insights into the origin of the large-area, non-local, collective ferroelastic switching behavior with respect to co-existing energetically degenerate nanodomain variants are provided.

\section{Results}

Epitaxial growth of $\mathbf{P b T i O}_{3}$ thin films. $70 \mathrm{~nm} \mathrm{PbTiO}_{3} / 20 \mathrm{~nm}$ $\mathrm{Ba}_{0.5} \mathrm{Sr}_{0.5} \mathrm{RuO}_{3} / \mathrm{SmScO}_{3}(110)_{O}$ heterostructures were deposited by pulsed-laser deposition ("Methods") ${ }^{36}$. The nominal misfit strain between the $\mathrm{PbTiO}_{3}$ film and substrate, controlled to be near a critical tensile strain (Supplementary Fig. 1), is close to the middle of the critical misfit strains of $0.2 \%$ (below $0.2 \%, c / a$ domain structures are favored) and $0.8 \%$ (above $0.8 \%, a_{1} / a_{2}$ domain structures are favored) where a nearly equal coexistence of the $c / a$ - and $a_{1} / a_{2}$-domain variants is expected ${ }^{36}$. X-ray diffraction studies and reciprocal space mapping analysis (Supplementary Figs. 2 and 3) reveal the presence of high-quality, epitaxial growth of the single-phase $\mathrm{PbTiO}_{3}$ films with $a$ and $c$ domains. Cross-section, bright-field transmission electron microscopy (TEM) (Fig. 1a) and plan-view high-angle annular dark-field-scanning transmission electron microscopy (HAADFSTEM) (Fig. 1b) studies confirm the presence of $c / a$-domain variants with domain walls parallel to the $[111]_{O}$ (i.e., [101] in pseudocubic indices), and $a_{1} / a_{2}$-domain variants with domain walls along the $[1 \overline{1} 1]_{O}$ and $[1 \overline{1} \overline{1}]_{O}$ (i.e., $[110]$ and $[1 \overline{1} 0]$ in pseudocubic indices, respectively) and domain widths of $\sim 30 \mathrm{~nm}$ (Fig. 1b). Note that the $a_{1} / a_{2}$ domains are obscured in the crosssection TEM since the $a_{1} / a_{2}$ domain walls are not aligned along the projected zone axis ${ }^{41}$. Likewise, the $c / a$ domains are obscured in the plan-view imaging as their domain walls run through the thickness of the sample. Local strain fields are assessed by geometric phase analysis (GPA, Gatan Digital Micrograph) and visualized using the Gatan Digital Micrograph software ${ }^{42}$. As compared with the lattice parameters of the bulk counterpart, the in-plane strain (Fig. 1c) and lattice rotation (Fig. 1d) maps extracted from the HAADF-STEM lattice image of the selected area (red square, Fig. $1 \mathrm{~b}$ ) show near perfect periodic elastic fields within the $a_{1} / a_{2}$ domains (head-to-tail domain structures, Fig. 1d). Furthermore, the lattice rotation map reveals relatively large fluctuations especially near the needle-shaped domains (yellow square, Fig. 1d), suggesting a high degree of structural softness (Fig. 1d) ${ }^{31}$. Previous studies in ferroelectric $\mathrm{KH}_{2} \mathrm{PO}_{4}$ crystals found that the interaction between such needle-shaped domain tips is long range ${ }^{43,44}$. The observed needle domains in these $\mathrm{PbTiO}_{3}$ films may also enhance the mobility of domain walls and long-range interactions ${ }^{42,45}$. The co-existing domain structure is further confirmed by piezoresponse force microscopy 

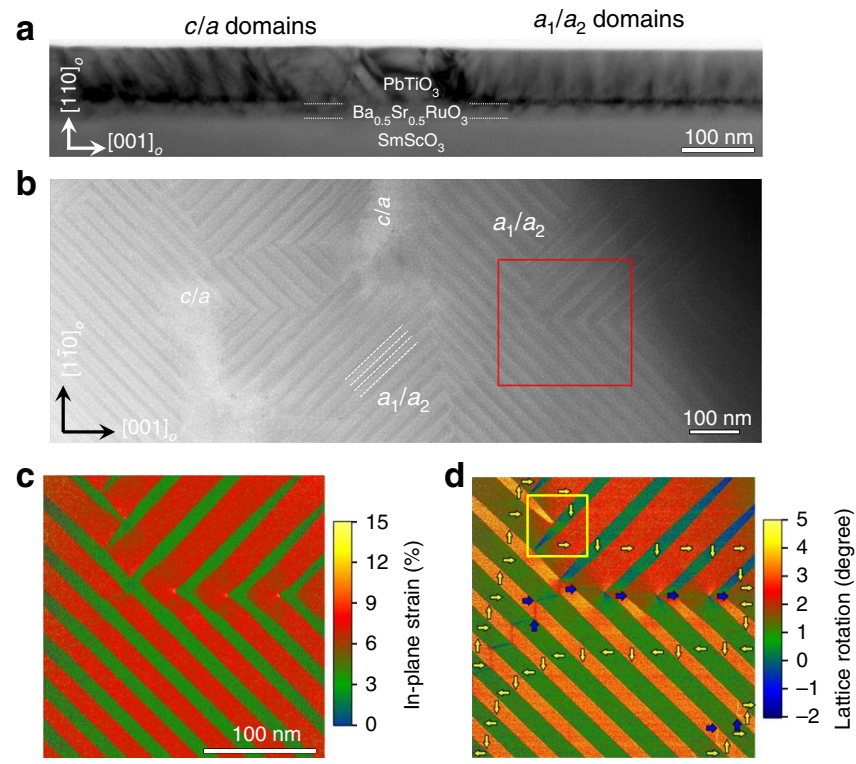

Fig. 1 Domain structures of 70-nm-thick $\mathrm{PbTiO}_{3}$ films grown on $\mathrm{Ba}_{0.5} \mathrm{Sr}_{0.5} \mathrm{RuO}_{3} / \mathrm{SmScO}_{3}(110)_{0}$. a Cross-sectional transmission electron microscopy (TEM) image of the heterostructures where the white dashed lines mark the $\mathrm{PbTiO}_{3} / \mathrm{Ba}_{0.5} \mathrm{Sr}_{0.5} \mathrm{RuO}_{3}$ and $\mathrm{Ba}_{0.5} \mathrm{Sr}_{0.5} \mathrm{RuO}_{3} / \mathrm{SmScO}_{3}$ interfaces. b Plan-view high-angle annular dark field-scanning transmission electron microscopy (HAADF-STEM) image of the heterostructure where the dashed lines indicate the $a_{1} / a_{2}$ domain walls. c In-plane strain map $\left(\varepsilon_{\mathrm{xx}}\right)$ and $\mathbf{d}$ lattice rotation map $(\omega)$ extracted from the HAADF-STEM lattice image via geometrical phase analysis (GPA) for the area in the red square in (b). The yellow arrows indicate the head-to-tail structures and the blue arrows indicate $180^{\circ}$ domain walls. The lattice rotation at $180^{\circ}$ domain walls can be used to identify the polarization directions beside the domain walls. Yellow-border square in (d) reveals relatively large fluctuations especially near the needle-shaped domains

(PFM) studies (Supplementary Fig. 4). The well-ordered $c / a$ and $a_{1} / a_{2}$ nanodomains observed in the $\mathrm{PbTiO}_{3}$ thin films should naturally have strong elastic interactions between neighboring domains due to the large crystal anisotropy, which, in turn, may facilitate collective switching behavior in the ferroelectric/ferroelastic system.

Reversible control of ferroelastic switching by out-of-plane electric fields. Earlier reports on (001)-oriented tetragonal ferroelectric thin films, such as $\mathrm{PbTiO}_{3}$ and $\mathrm{PbTi}_{0.8} \mathrm{Zr}_{0.2} \mathrm{O}_{3}$ films grown on $\mathrm{SrTiO}_{3}(001)$ substrates ${ }^{14}$, indicate that it is challenging for large-area ferroelastic switching between $a$ and $c$ domains using out-of-plane electric fields because the compressive strain imposed by the substrate strongly (elastically) favors $c$ domains. If the energy barrier between the in- and out-of-plane polarized domains is made small, however, by, for example, tuning the misfit strain, ferroelastic switching could occur ${ }^{36}$. Furthermore, large-area, even non-local, ferroelastic switching could be enabled when the film is tuned close to the domain-structure boundary.

To explore this idea, we first applied different out-of-plane DC voltages using a PFM tip in a representative $1.2 \times 1.2 \mu \mathrm{m}$ area (Fig. 2a-e). Upon poling the entire area with a $-4 \mathrm{~V}$ bias applied through the tip, the majority of the $a_{1} / a_{2}$ domains in the as-grown state (with weak out-of-plane PFM response, Fig. 2a) reorganize into $c / a$ domains (with strong out-of-plane PFM response and upward pointing $c$ domains, Fig. $2 b$ ). In order to investigate the reversibility of the ferroelastic switching, stepwise positive bias voltages were applied in an attempt to switch the induced $c / a$ domain structures back to $a_{1} / a_{2}$-domain structures. At a $+2 \mathrm{~V}$ applied bias, the $c / a$-domain structures begin to switch back into $a_{1} / a_{2}$ domains (with low vertical amplitude, Fig. $2 \mathrm{c}$ ). By $+2.5 \mathrm{~V}$ applied bias, the upward pointing $c$-domain regions gradually disappear (Fig. 2d). Upon further increasing the poling voltage to $+3 \mathrm{~V}$, the $a_{1} / a_{2}$-domain structures are again interconverted back to $c / a$-domain structures, but this time with the $c$ variants poled downward (Fig. 2e). As revealed above, the domain evolution under stepwise voltage suggests that ferroelastic switching readily occurs and is reversible as per the process described herein (Fig. 2f). Although $c / a$ domains can be switched back to $a_{1} / a_{2}$ domains by application of large in-plane voltage (which also requires lithographic patterning) as illustrated in earlier studies ${ }^{36}$, it is interesting to note that one can also switch the $c / a$ domains back into $a_{1} / a_{2}$ domains step-by-step with a small and simple out-of-plane electric field. This further suggests the rather small energy barrier between these domain structures in the films.

Mechanical force-induced non-local ferroelastic switching. The above demonstration of reversible $a_{1} / a_{2}$ to $c / a$ ferroelastic switching by purely out-of-plane electric fields, however, is found to be localized, probably due to weak electrostatic interactions. To trigger a larger area non-local switching, an appropriate driving force, something more influential than electrostatics, is required. In this regard, compared with electric field, mechanical force has the potential to provide the long-range elastic interaction necessary to drive these effects ${ }^{46}$. It is important to note that several studies of mechanical force-induced domain switching in ferroelectric thin films (typically due to flexoelectric effects) have been reported ${ }^{47,48}$. Only local response to the applied mechanical force, however, was observed in those studies. This is again due to the large compressive misfit strain which likely favors $c$ domains and thus hampers ferroelastic switching. To enable collective domain switching, the system should be carefully tuned to the brink of a structural instability, as we have achieved here by delicately controlling the epitaxial strain.

We apply a point array of force using an atomic force microscope Fmap ("Methods") on an area of the film which possesses a majority of $a_{1} / a_{2}$ domain areas (Supplementary Fig. 4). The force mapping is completed in a $2 \times 2$ array of points (the tip radius is only $\sim 25 \mathrm{~nm}$ ) at the corners of a $1 \times 1 \mu \mathrm{m}$ area within a $2 \times 2 \mu \mathrm{m}$ scanned area (Fig. $3 \mathrm{a}-\mathrm{d}$ ). After the application of a setpoint with voltage of $2 \mathrm{~V}$, corresponding to a force of $\sim 600 \mathrm{nN}$ ("Methods"), to the noted positions, a dramatic change in the domain structure occurs even outside the tip-sample contact area and appears in both the topography (Fig. 3a, b) and out-of-plane PFM amplitude (Fig. $3 \mathrm{c}, \mathrm{d}$ ) images. More specifically, it is found that the majority of the initial $a_{1} / a_{2}$-domain structures across the entire scanned area are converted to $c / a$ domain structures. We note that the changes can extend across nearly the entire $2 \times 2 \mu \mathrm{m}$ scanned area (Fig. $3 \mathrm{~b}$, d, and Supplementary Fig. 5). These changes are made more evident by extracting the evolution of the surface morphology and out-ofplane PFM amplitude (dashed lines, Fig. 3a-d) across these switched areas (which is outside the tip-sample contact region). After applying the local mechanical force, the average height and out-of-plane PFM amplitude of the switched area are $\sim 800 \mathrm{pm}$ and $\sim 300 \mathrm{pm}$ higher than those of the as-grown $a_{1} / a_{2}$ domains (Fig. 3e, f), respectively; clearly demonstrating that large-area, non-local ferroelastic switching occurs (Supplementary Figs. 6-8).

Phase-field simulations of the domain switching under tipinduced mechanical force. To understand the large non-local response observed herein, we employed phase-field simulations ("Methods") to model the domain switching under tip-induced mechanical force ${ }^{49}$. Since the observed non-local response occurs 

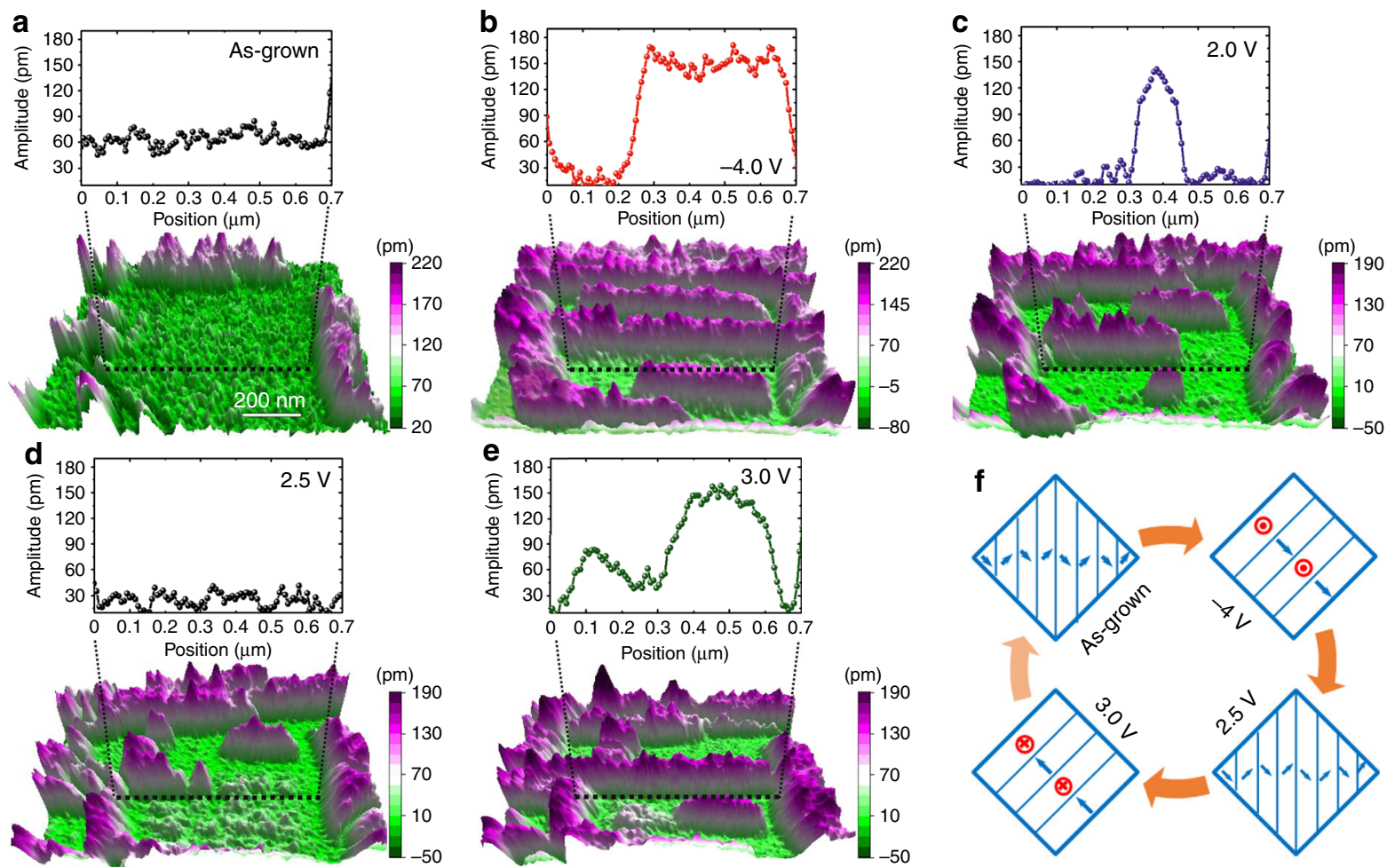

Fig. 2 Ferroelastic switching via out-of-plane electric field poling. 3D representation of the out-of-plane piezoresponse force microscopy (PFM) amplitude and corresponding line trace along the noted dashed lines in (a), as-grown state, $\mathbf{b}$ after being poled upward via a $-4 \mathrm{~V}$ bias, $\mathbf{c}$ after an applied $+2 \mathrm{~V}$ bias, $\mathbf{d}$ after an applied $+2.5 \mathrm{~V}$ bias, and $\mathbf{e}$ after further increasing bias to $+3 \mathrm{~V}$. $\mathbf{f}$ Schematic illustration of the domain-structure evolution under stepwise electric field
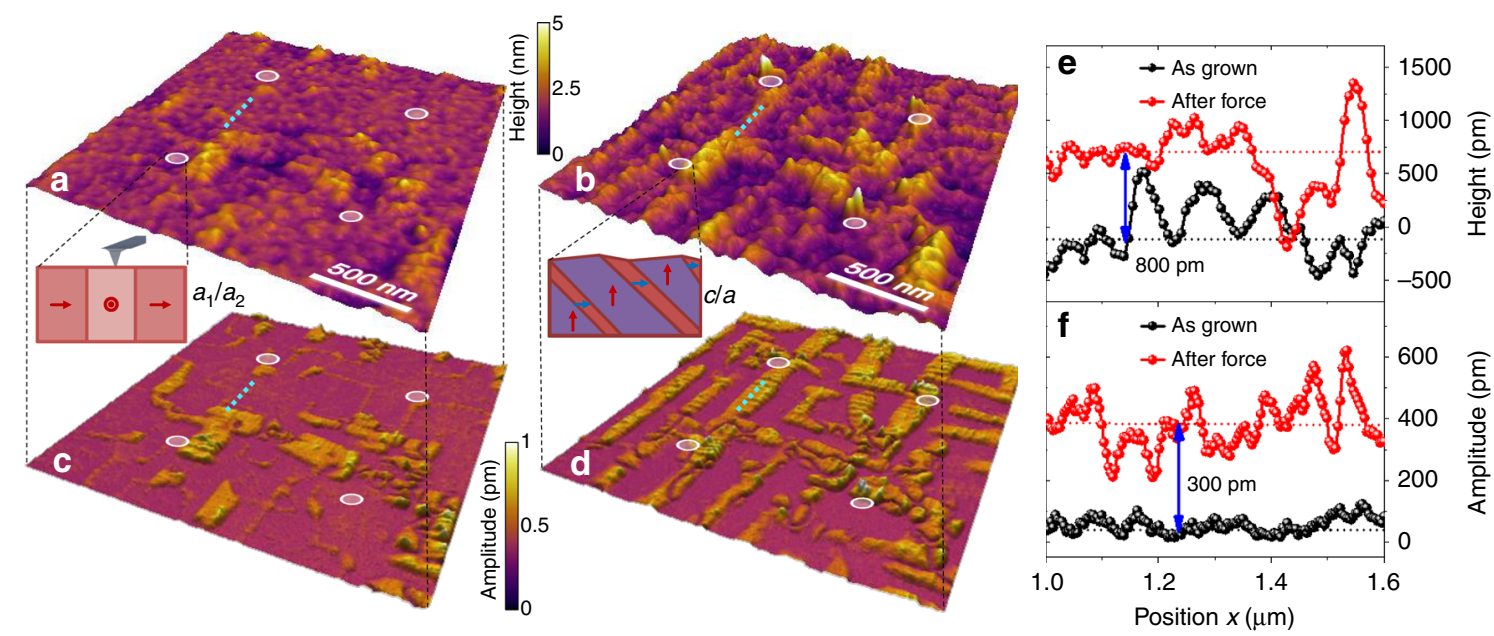

Fig. 3 Mechanical force-induced non-local, large-area ferroelastic switching. The force mapping is completed in a $2 \times 2$ array of points (the tip radius is $\sim 25 \mathrm{~nm}$ ) at the corners of $\mathrm{a} 1 \times 1 \mu \mathrm{m}$ area within a $2 \times 2 \mu \mathrm{m}$ scanned area. Topographic images of the films $\mathbf{a}$ before and $\mathbf{b}$ after the application of local fourpoint force as noted by four shaded circles. Out-of-plane piezoresponse force microscopy (PFM) images in c as-grown state and $\mathbf{d}$ after the four-point force mapping. A dramatic change in the domain structure occurs outside the tip-sample contact area and appears in both the topography $\mathbf{b}$ and out-of-plane PFM amplitude $\mathbf{d}$ images after the application of $\sim 600 \mathrm{nN}$ force to the noted positions. Corresponding line profile changes in $\mathbf{e}$ surface height and $\mathbf{f}$ out-ofplane PFM amplitude as indicated by the dashed lines in (a-d). The average height and out-of-plane PFM amplitude of the switched area are $\sim 800$ pm and $\sim 300 \mathrm{pm}$ higher than those of the as-grown $a_{1} / a_{2}$ domains, respectively

in the areas where $a_{1} / a_{2}$ domains dominate, we start from a quasi-stable state of $a_{1} / a_{2}$ domains under a $0.5 \%$-strain state (time step 0, Fig. 4a). Upon application of the four-point $600 \mathrm{nN}$ tip force in a $2 \times 2$ array, non-local $a_{1} / a_{2}$ to $c / a$ switching gradually occurs which eventually penetrates through the entire film thickness (with $c / a$ walls oriented $45^{\circ}$ in the cross-section $x$-z plane) (Fig. 4), consistent with the experimental results. After applying the local mechanical force, the initial $a_{1} / a_{2}$ domains beneath the probe tip remain unchanged because the large, tipinduced out-of-plane compression would favor $a$ domains with 

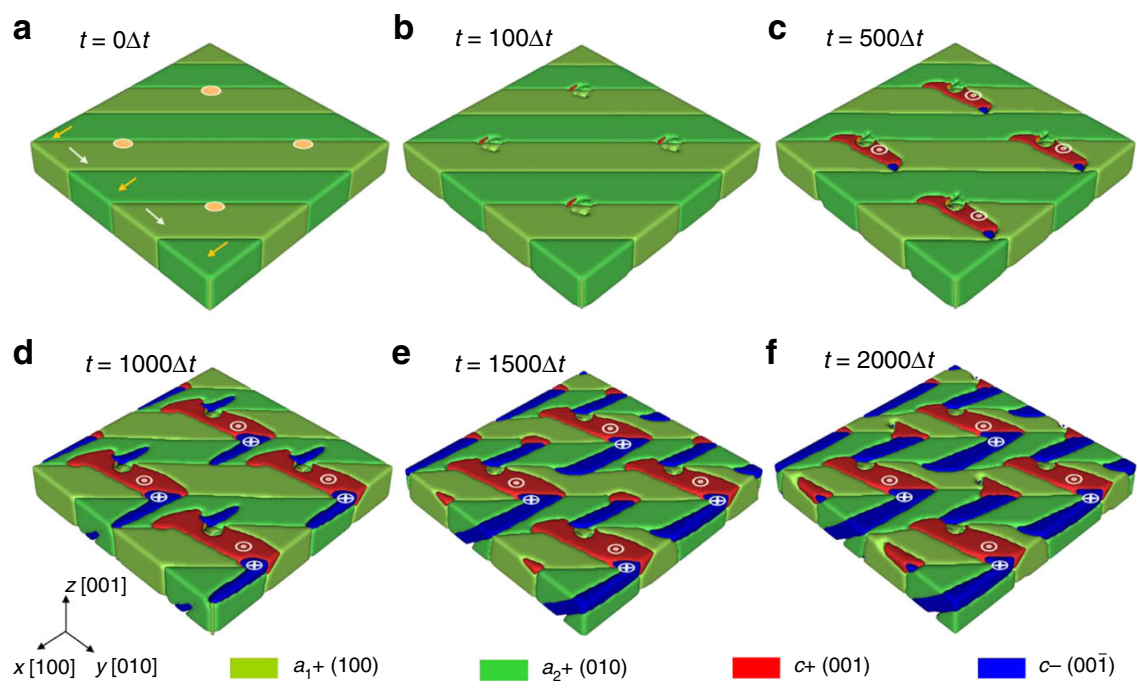

Fig. 4 Phase-field simulations of mechanical force-induced domain evolution. The films with $a_{1} / a_{2}$ domains are subjected to $0.5 \%$ tensile strain. a Asgrown $a_{1} / a_{2}$ state at $t=0 \Delta t$ with illustration of probe tip force locations as noted by four circles, and arrows indicate the polarization directions of $a_{1} / a_{2}$ domains, $\mathbf{b} t=100 \Delta t$ where $c$ domains emerge around the tip-sample contact area, $\mathbf{c} t=500 \Delta t$ where more $c+$ domains (red area, polarization up, noted with $\odot$ ) are formed and $c$ domains (blue area, polarization down, noted with $\oplus$ ) adjacent $c+$ domains begin to emerge, $\mathbf{d}, t=1000 \Delta t$ where $c$ domains penetrate all the way down to the bottom, $\mathbf{e}, t=1500 \Delta t$ and $\mathbf{f}, t=2000 \Delta t$ where more $c$ domains begin to form, resulting $c / a$ domains structures

in-plane polarization (Supplementary Fig. 9). The lattice of asgrown, in-plane polarized a domains under the tip-induced pressure will slightly expand along the in-plane direction ${ }^{50}$. To match the epitaxial tensile strain, $c$ domains are formed adjacent to the $a$ domains to locally decrease the average in-plane lattice parameter, resulting in head-to-tail c/a-domain structures. Moreover, near the tip-sample contact area, strain-gradient induced, out-of-plane flexoelectric fields would also favor the formation of $c$ domains (Supplementary Fig. 10) ${ }^{51}$. Under these combined effects, more $c$ domains emerge at the top surface of the film near the tip-sample contact area (Fig. $4 \mathrm{~b}$, c). Eventually, $c$ domains penetrate all the way across the film over a large area to maintain the lowest total free energy (Fig. $4 \mathrm{~d}-\mathrm{f}$ ). Based on the phase-field simulations, the Landau free energy and electrostaticenergy density both decrease during the formation of the $c$ domains, while the gradient-energy density increases due to the formation of domain walls (Supplementary Fig. 11). This domain propagation is also evident in the in-plane tensile strain fields, which expand gradually into a large area corresponding to the formation of the $c$ domains (Supplementary Fig. 12). Again, to match the epitaxial tensile strain, $a$ domains are favored next to $c$ domains, then, followed with $a$ domains. Since the surrounding $a_{1} / a_{2}$ domains are already relatively unstable under the tensile stress surrounding the probe tip, the system is driven into successive switching with energetically favorable head-to-tail $c / a$ configurations co-existing with remaining $a_{1} / a_{2}$ domains until a new energy equilibrium is reached. Since the structure prefers to maintain head-to-tail domain structures to minimize the total energy, the change of domain structures in a local area will release energy and eventually propagate across long distances, leading to collective switching.

\section{Discussion}

Such a collective domain switching process is strongly related to the potential energy barrier between the two domain-structure variants. Based on Landau-type phenomenological theory for polydomain structures ${ }^{38}$, the calculated Landau free-energy densities of the $a_{1} / a_{2}$ and $c / a$ domain patterns are equal at the critical strain of $\sim 0.46 \%$ at $300 \mathrm{~K}$; however, at high temperature, such as $500 \mathrm{~K}$, are about $-22 \mathrm{MJ} / \mathrm{m}^{3}$ and $-19 \mathrm{MJ} / \mathrm{m}^{3}$, respectively, with an energy difference of only $\sim 3 \mathrm{MJ} / \mathrm{m}^{3}$ (Supplementary Fig. 13). The transition barrier between the $a_{1} / a_{2}$ and $c / a$-domain patterns is much lower than the energy required for the polarization switch, such as the $c^{+}$to $c^{-}$, of about $48 \mathrm{MJ} / \mathrm{m}^{3}$ (Supplementary Fig. 13). Such a small energy barrier between the $a_{1} / a_{2^{-}}$and $c / a$-domain variants gives the possibility of domain switching propagation. Since the initial domains formed in the asgrown heterostructures are dominated by the $a_{1} / a_{2}$ domains due to the relatively lower free-energy potential, external stimuli that overcomes the initial energy barrier will force the system to reconfigure into a more favorable state (i.e., co-existing $a_{1} / a_{2}$ and c/a domains). Specifically, the mechanical force applied by the probe tip can gently lower the energy barrier and drive the system to a final state favoring a domain pattern with the coexistence of $a_{1} / a_{2}$ and $c / a$ domains.

This is further supported by phase-field simulations of similar films under different strain states. When the misfit tensile strain is $<0.2 \%$, the $c / a$ domains dominate and the $a_{1} / a_{2}$-domain structure is unstable even before applied tip forces (Supplementary Figs. $14 \mathrm{a}-\mathrm{c}$ ). For films under misfit strains $>0.3 \%$, however, the pure $a_{1} / a_{2}$-domain structures are in quasi-steady state (Supplementary Fig. 14d-e). We also note that the thickness effect in the phase-field simulation is small (Supplementary Fig. 15). In a small range of misfit strains $(0.3-0.5 \%)$, non-local response is triggered upon the application of tip pressures, accompanied with a sudden decrease of the total free energy, indicating that the newly formed co-existing $c / a$ and $a_{1} / a_{2}$ domains have lower total free energy than the original $a_{1} / a_{2}$-domain structures (Fig. 5a). It is found that the $a_{1} / a_{2}$ domains become more favorable with increasing tensile strain, and thus the initial total free energy density $(t=0)$ decreases with increasing misfit strain $\left(u_{\mathrm{m}}\right)$, and the decrease of the total average free-energy density becomes smaller with subsequent collective switching. Such collective switching disappears upon further increasing the misfit strain. For example, films under a tensile strain of $1 \%$ have an equilibrium state of $a_{1} / a_{2}$ domains and only subtle changes exist near the tip contact area (Fig. 5b). Ultimately, both the experiments and phase-field simulations reveal that the delicately balanced elastic field in such systems with co-existing domains is crucial for the 
a

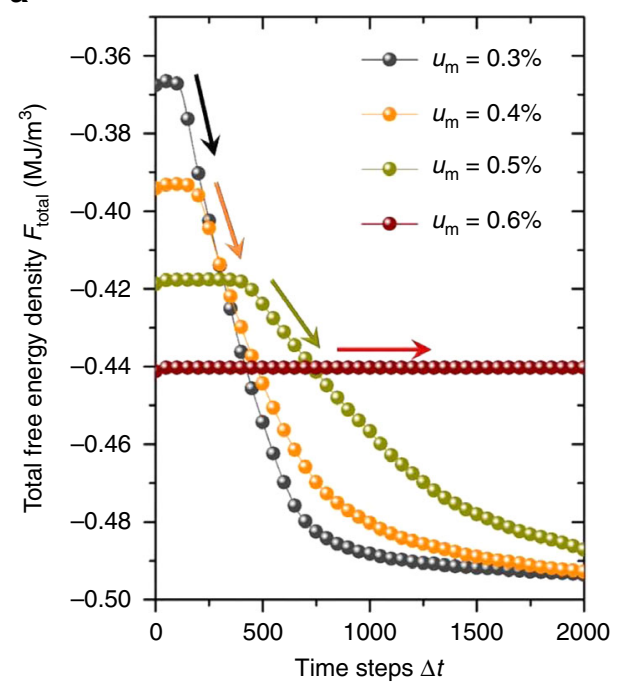

b $\quad u_{m}=1.0 \%$

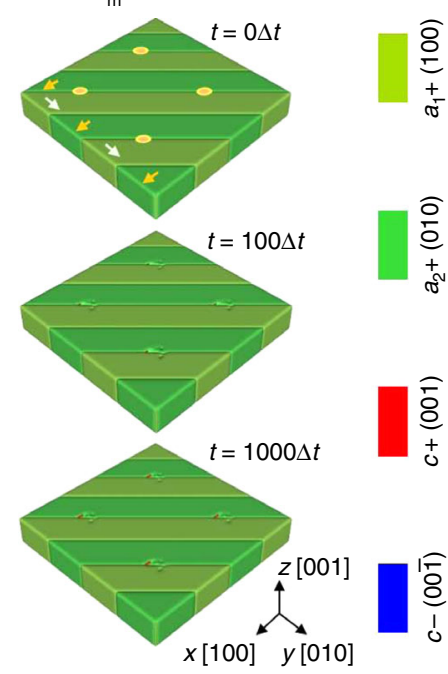

Fig. 5 Phase-field simulation of domain evolution under various strain states. a Total free-energy density changes after the probe tip-induced force exerted on an initially pure $a_{1} / a_{2}$ domain structure of the films subjected to various tensile strains ranging from $+0.3 \%$ to $0.6 \%$. The corresponding arrows indicate the steepness of the energy changes. $\mathbf{b}$ Phase-field simulations of mechanical force-induced domain evolution of the films with $a_{1} / a_{2}$ domains subjected to $1 \%$ tensile strain. Probe tip force locations are noted by four circles on the film surface

large response since the elastic field in the film can be easily rebuilt and extended under external perturbation.

In summary, we have successfully demonstrated a long-range, non-local ferroelastic switching behavior in tensile-strained $\mathrm{PbTiO}_{3}$ thin films on $\mathrm{SmScO}_{3}$ (110) substrates wherein $a_{1} / a_{2}$ and $c / a$-domain structures coexist. Due to the low-energy barrier between the energetically degenerate periodic domain structures, an exotic mechanical force-induced ferroelastic switching with an area much larger than the direct contacting points is observed, accompanied by a large change in topography and piezoelectric response. Phase-field simulations reveal that the nearly energetically degenerate nature of the different domain states is the cause of the delicately balanced elastic field, which is responsible for the large response since the elastic field can be easily rebuilt and extended under external perturbations. Our results pave a new way for possible applications in sensitive mechanical sensors and switches by manipulating the ferroelastic switching behavior of ferroelectric thin films.

\section{Methods}

Thin film synthesis. Heterostructures were grown via pulsed-laser deposition using a $\mathrm{KrF}$ excimer laser ( $248 \mathrm{~nm}$, Compex, Coherent). Epitaxial 70-nm-thick $\mathrm{PbTiO}_{3}$ films were grown on $20 \mathrm{~nm} \mathrm{Ba}{ }_{0.5} \mathrm{Sr}_{0.5} \mathrm{RuO}_{3} / \mathrm{SmScO}_{3}(110)_{O}$ substrates (CrysTec GmbH, Germany) from ceramic targets. The $\mathrm{Ba}_{0.5} \mathrm{Sr}_{0.5} \mathrm{RuO}_{3}$ layer was grown at a temperature of $750{ }^{\circ} \mathrm{C}$ in a dynamic oxygen pressure of $20 \mathrm{mTorr}$ at a laser repetition rate of $3 \mathrm{~Hz}$ and a laser fluence of $1.9 \mathrm{~J} / \mathrm{cm}^{2}$. The $\mathrm{PbTiO}_{3}$ layer was grown at a temperature of $675^{\circ} \mathrm{C}$, in a dynamic oxygen pressure of $50 \mathrm{mTorr}$ at a laser repetition rate of $10 \mathrm{~Hz}$, and a laser fluence of $1.9 \mathrm{~J} / \mathrm{cm}^{2}$. Following growth, the heterostructures were cooled down to room temperature at a rate of $20^{\circ} \mathrm{C} / \mathrm{min}$, in a static oxygen pressure of 700 Torr.

Structural characterization via X-ray diffraction. $\theta-2 \theta$ measurements were carried out using a Panalytical X'pert Pro diffractometer with $\mathrm{Cu} K_{\alpha_{1}}$ radiation (wavelength $\lambda$ of $1.54056 \AA$ ). X-ray reciprocal space mapping studies were carried out at the Sector 33-BM-C beamline of the Advanced Photon Source, Argonne National Laboratory.

Scanning transmission electron microscopy (STEM). Cross-sectional and planview samples were prepared by slicing, grinding, dimpling, and finally ion-milling using a Gatan PIPS, while the plan-view samples were milled only from the substrate side. The HAADF-STEM images were obtained by using a Titan G2 60e300 microscope with a high-brightness field emission gun and double aberration (Cs) correctors from CEOS operating at $300 \mathrm{kV}$. Strain fields were deduced by using custom plugins of GPA for the software Gatan Digital Micrograph. The visualization of strains and lattice rotations were carried out using the Gatan Digital Micrograph software.

PFM studies and force mapping. The PFM studies were carried out on a MFP3D, (Asylum Research) in DART mode using Ir/Pt-coated conductive tips (Nanosensor, PPP-NCLPt with spring constant $k_{\text {tip }}$ of $\sim 3 \mathrm{Nm}^{-1}$ and AmpInvOLS of $100.55 \mathrm{~nm} / \mathrm{V})$. The tip spring constant $k_{\text {tip }}$ was calibrated by using a quartz plate

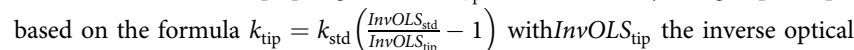
lever sensitivity (with units of $\mathrm{nm} / \mathrm{V}$ ) for the cantilever under test measured on a very stiff surface, and $\operatorname{InvOLS}$ std the same quantity measured on a compliant surface with spring constant $k_{\text {std }}$. We note that the software Igor Pro 6.37 (Asylum Research) can determine the cantilever's spring constant based on the equipartition theorem following a 3-step procedure in the Manual of spring constant determination (MFP-3D ${ }^{\mathrm{m}}$ Procedural Operation 'Manualette' Version 10 (v080501; Igor $6.04 \mathrm{~A}$ )). The applied driving voltage (relative trigger point) of $2 \mathrm{~V}$ corresponds to the force of $600 \mathrm{nN}$ with the tip spring constant of about $3 \mathrm{Nm}^{-1}$ (Nanosensor PPP-EFM) based on the formula $F=k_{\text {tip }} \cdot A m p I n v O L S \cdot V$. We note that the force mapping uses direct current (DC) voltage, while the PFM imaging scan uses alternating current (AC) driving voltage of $1 \mathrm{~V}$ in dual AC resonance tracking (DART) mode with smaller contact force.

Force mapping was completed with a $2 \times 2$ array of points on the corners of the mapping area. First, the tip was lifted to a distance of $1.5 \mu \mathrm{m}$ above the film surface, then fast driven down to the point with a velocity of $2.98 \mu \mathrm{m} / \mathrm{s}$. After a dwell time set as $0.99 \mathrm{~s}$, the tip was then lifted and moved to the second point in the order of from top-to-bottom and from left-to-right in each row with velocity of $1.5 \mu \mathrm{m} / \mathrm{s}$.

Phase-field simulations. In the phase-field simulations of the domain switching under mechanical tip pressure, we use polarization vector $\mathbf{P}=\left(P_{1}, P_{2}, P_{3}\right)$ as the order parameter to describe the ferroelectric state in the $\mathrm{PbTiO}_{3}$ thin film. The temporal evolution of $P_{i}(i=1-3)$ is calculated by minimizing the total free energy $F$ with respect to $P_{i}$ via numerically solving the time-dependent Landau-Ginzburg-Devonshire (LGD) equations ${ }^{37}$,

$$
\frac{\partial P_{i}(\mathbf{x}, t)}{\partial t}=-L \frac{\delta F}{\delta P_{i}(\mathbf{x}, t)},(i=1 \sim 3)
$$

where $\mathbf{x}$ is the spatial position (with $x-, y_{-}, \mathrm{z}$ - axes along the [100], [010], and [001] Cartesian coordinate directions), $t$ is the time, $L$ is the kinetic coefficient related to the domain wall mobility. The total free energy $F$ of the PTO thin film includes the Landau, gradient, elastic, electrostatic, and flexoelectric energies, which is written as,

$$
F=\int_{V}\left[f_{\text {lan }}\left(P_{i}\right)+f_{\text {grad }}\left(\nabla P_{i}\right)+f_{\text {elas }}\left(P_{i}, \varepsilon_{i j}\right)+f_{\text {elec }}\left(P_{i}, E_{i}\right)+f_{\text {flexo }}\left(P_{i}, \varepsilon_{k l}, \nabla P_{i}, \nabla \varepsilon_{k l}\right)\right] d V
$$

where $V$ is the total volume of the system, $\varepsilon_{i j}$ and $E_{i}$ denote the components of strain and electric fields, $\nabla$ is the gradient operator. Detailed expressions of each free-energy density can be found in the Ref. ${ }^{47}$. Equation (1) is numerically solved using a semi-implicit spectral method ${ }^{52}$ based on a $3 D$ geometry sampled on a 
$128 \Delta x \times 128 \Delta y \times 32 \Delta z$ system size, with $\Delta x=\Delta y=\Delta z=1.0 \mathrm{~nm}$. The thickness of the film, substrate, and air are $20 \Delta z, 10 \Delta z$, and $2 \Delta z$, respectively. The temperature is $T=25^{\circ} \mathrm{C}$, and an isotropic relative dielectric constant $\left(\kappa_{\mathrm{ii}}\right)$ is chosen to be 50 . The gradient-energy coefficients are set to be $G_{11} / G_{110}=0.6$, while

$G_{110}=1.73 \times 10^{-10} \mathrm{C}^{-2} \mathrm{~m}^{4} \mathrm{~N}$. The Landau coefficients, electrostrictive coefficients, and elastic-compliance constants are collected from Ref. ${ }^{53}$. The flexoelectric energy can be written as ${ }^{54}$

$$
f_{\text {flexo }}=\frac{F_{i j k l}}{2}\left(\varepsilon_{i j} \frac{\partial P_{k}}{\partial x_{l}}-P_{k} \frac{\partial \varepsilon_{i j}}{\partial x_{l}}\right)
$$

where, $F_{i j k l}(i=1 \sim 3)$ are the flexoelectric coupling coefficients. In our previous work we found that the longitudinal $\left(F_{11}\right)$, shear $\left(F_{12}\right)$ and transverse $\left(F_{44}\right)$ flexoelectric coefficients could affect the polarization tilt near the domain wall/substrate junctions, where $F_{\mathrm{ij}}$ 's are the three independent flexoelectric coupling coefficients in the cubic system using Voigt notation, i.e., $F_{11}=F_{1111}, F_{12}=F_{1122}$, and $F_{44}=2 F_{1221}$. Due to the uncertainties of the magnitude of $F_{\mathrm{ij}}$ 's, we use $F_{11}=1.0 \times 10^{-11}\left(\mathrm{Vm}^{2} \mathrm{~N}^{-1}\right)$ and assume $F_{12}=F_{44}=0$ for simplicity based on literature ${ }^{52}$. We model the scanningprobe tip as a spherical indenter on the film surface, and define the normal stress distribution on top surface based on the Hertzian model,

$$
\sigma_{33}^{\mathrm{tip}}(r)=\left\{\begin{array}{cc}
-\frac{3 F}{2 \pi a^{2}} \sqrt{1-\frac{r^{2}}{a^{2}}} & (r \leq a) \\
0.0 & (r \geq a)
\end{array}\right.
$$

in which $F$ is the mechanical load, $a$ is the radius of the tip-sample contact area, and $r=\sqrt{\left(x-x_{0}\right)^{2}+\left(y-y_{0}\right)^{2}}$ is the distance from any points $(x, y)$ inside the contact area to the tip center $\left(x_{0}, y_{0}\right)$.

\section{Data availability}

The data that support the findings of this study are available from the corresponding author upon reasonable request.

Received: 16 November 2018 Accepted: 17 July 2019

Published online: 02 September 2019

\section{References}

1. Damjanovic, D. Ferroelectric, dielectric and piezoelectric properties of ferroelectric thin films and ceramics. Rep. Prog. Phys. 61, 1267-1324 (1998).

2. Tagantsev, A. K., Cross, L. E. \& Fousek, J. Ferroelectric Domains in Ferroelectric Crystals and Thin Films. (Springer, 2010).

3. Nagarajan, V. et al. Dynamics of ferroelastic domains in ferroelectric thin films. Nat. Mater. 2, 43 (2003).

4. Balke, N. et al. Deterministic control of ferroelastic switching in multiferroic materials. Nat. Nanotech. 4, 868-875 (2009).

5. Ren, X. Large electric-field-induced strain in ferroelectric crystals by pointdefect-mediated reversible domain switching. Nat. Mater. 3, 91-94 (2004).

6. Lee, K. \& Baik, S. Ferroelastic domain structure and switching in epitaxial ferroelectric thin films. Annu. Rev. Mater. Res. 36, 81-116 (2006).

7. Gao, P. et al. Ferroelastic domain switching dynamics under electrical and mechanical excitations. Nat. Commun. 5, 3801 (2014).

8. Heron, J. et al. Deterministic switching of ferromagnetism at room temperature using an electric field. Nature 516, 370-373 (2014).

9. Baek, S. et al. Ferroelastic switching for nanoscale non-volatile magnetoelectric devices. Nat. Mater. 9, 309-314 (2010).

10. Lahtinen, T. H. et al. Pattern transfer and electric-field-induced magnetic domain formation in multiferroic heterostructures. Adv. Mater. 23, 3187-3191 (2011).

11. Calderon-Moreno, J. M. Stress induced domain switching of PZT in compression tests. Mater. Sci. Engin.: A 315, 227-230 (2001).

12. Webber, K. G. et al. Temperature-dependent ferroelastic switching of soft lead zirconate titanate. Acta Mater. 57, 4614-4623 (2009).

13. Garcia, J. et al. Evaluation of domain wall motion in lead zirconate titanate ceramics by nonlinear response measurements. J. Appl. Phys. 103, 054108 (2008).

14. Ganpule, C. et al. Role of 90 domains in lead zirconate titanate thin films. Appl. Phys. Lett. 77, 292-294 (2000).

15. Chu, M. W. et al. Impact of misfit dislocations on the polarization instability of epitaxial nanostructured ferroelectric perovskites. Nat. Mater. 3, 87-90 (2004).

16. Nagarajan, V. et al. Misfit dislocations in nanoscale ferroelectric heterostructures. Appl. Phys. Lett. 86, 192910 (2005).

17. Matzen, S. et al. Super switching and control of in-plane ferroelectric nanodomains in strained thin films. Nat. Commun. 5, 4415 (2014).

18. Feigl, L. et al. Compliant ferroelastic domains in epitaxial $\mathrm{Pb}(\mathrm{Zr}, \mathrm{Ti}) \mathrm{O}_{3}$ thin films. Appl. Phys. Lett. 104, 172904 (2014).
19. Khan, A. I. et al. Voltage-controlled ferroelastic switching in $\mathrm{Pb}\left(\mathrm{Zr}_{0.2} \mathrm{Ti}_{0.8}\right) \mathrm{O}_{3}$ thin films. Nano Lett. 15, 2229-2234 (2015).

20. Kim, K. E. et al. Configurable topological textures in strain graded ferroelectric nanoplates. Nat. Commun. 9, 403 (2018).

21. $\mathrm{Xu}, \mathrm{R}$. J. et al. Ferroelectric polarization reversal via successive ferroelastic transitions. Nat. Mater. 14, 79-86 (2015).

22. Wang, C. et al. Ferroelastic switching in a layered-perovskite thin film. Nat. Commun. 7, 10636 (2016).

23. Crassous, A. et al. Polarization charge as a reconfigurable quasi-dopant in ferroelectric thin films. Nat. Nanotech. 10, 614-618 (2015).

24. Cho, C.-R. et al. Dielectric and ferroelectric response as a function of annealing temperature and film thickness of sol-gel deposited $\mathrm{Pb}\left(\mathrm{Zr}_{0.52} \mathrm{Ti}_{0.48}\right)$ $\mathrm{O}_{3}$ thin film. J. Appl. Phys. 86, 2700-2711 (1999).

25. Nambu, S. \& Sagala, D. A. Domain formation and elastic long-range interaction in ferroelectric perovskites. Phys. Rev. B 50, 5838 (1994).

26. Aravind, V. R. et al. Correlated polarization switching in the proximity of a 180 degrees domain wall. Phys. Rev. B 82, 024111 (2010).

27. Bratkovsky, A. \& Levanyuk, A. Easy collective polarization switching in ferroelectrics. Phys. Rev. Lett. 85, 4614 (2000).

28. Li, J., Rogan, R., Üstündag, E. \& Bhattacharya, K. Domain switching in polycrystalline ferroelectric ceramics. Nat. Mater. 4, 776-781 (2005).

29. Bintachitt, P. et al. Collective dynamics underpins Rayleigh behavior in disordered polycrystalline ferroelectrics. Proc. Natl Acad. Sci. USA 107, 7219-7224 (2010)

30. Choudhury, S. et al. Correlation between number of ferroelectric variants and coercive field of lead ziconate titanate single crystals. Appl. Phys. Lett. 91, 032902 (2007).

31. Zeches, R. et al. A strain-driven morphotropic phase boundary in $\mathrm{BiFeO}_{3}$. Science 326, 977-980 (2009).

32. Ivry, Y. et al. Nucleation, growth, and control of ferroelectric-ferroelastic domains in thin polycrystalline films. Phys. Rev. B 86, 205428 (2012).

33. Le Rhun, G. et al. Piezoelectric response hysteresis in the presence of ferroelastic 90 domain walls. Appl. Phys. Lett. 90, 012908 (2007).

34. Morelli, A. et al. Deterministic switching in bismuth ferrite nanoislands. Nano Lett. 16, 5228-5234 (2016).

35. Vasudevan, R. K. et al. Exploring topological defects in epitaxial $\mathrm{BiFeO}_{3}$ thin films. ACS Nano 5, 879-887 (2011).

36. Damodaran, A. R. et al. Three-state ferroelastic switching and large electromechanical responses in $\mathrm{PbTiO}_{3}$ thin films. Adv. Mater. 29, 1702069 (2017).

37. Roitburd, A. L. Equilibrium structure of epitaxial layers. Phys. Stat. Sol. (a) 37, 329-339 (1976)

38. Roytburd, A. L., Ouyang, J. \& Artemev, A. Polydomain structures in ferroelectric and ferroelastic epitaxial films. J. Phys: Condens. Mater. 29, 163001 (2017).

39. Chen, L. Q. Phase-field models for microstructure evolution. Annu. Rev. Mater. Res. 32, 113-140 (2002).

40. Kukhar, V. et al. Polarization states of polydomain epitaxial $\mathrm{Pb}\left(\mathrm{Zr}_{1-x} \mathrm{Ti}_{x}\right) \mathrm{O}_{3}$ thin films and their dielectric properties. Phys. Rev. B 73, 214103 (2006)

41. Tang, Y. et al. On the benefit of aberration-corrected HAADF-STEM for strain determination and its application to tailoring ferroelectric domain patterns. Ultramicroscopy 160, 57-63 (2016).

42. $\mathrm{Li}, \mathrm{S}$. et al. Thickness-dependent $\mathrm{a}_{1} / \mathrm{a}_{2}$ domain evolution in ferroelectric $\mathrm{PbTiO}_{3}$ films. Acta Mater. 131, 123-130 (2017).

43. Bornarel, J. \& Lajzerowicz, J. Experimental evidence for the long-range interaction of domains in ferroelectric $\mathrm{KH}_{2} \mathrm{PO}_{4}$. J. Appl. Phys. 39, 4339-4341 (1968).

44. Schranz, W. Superelastic softening in perovskites. Phys. Rev. B 83, 094120 (2011).

45. Liu, Y. \& Li, J. Energetic analysis of ferroelectric domain patterns by equivalent inclusion method. J. Mater. Sci. 44, 5214-5224 (2009).

46. Frenkel, Y. et al. Anisotropic transport at the $\mathrm{LaAlO}_{3} / \mathrm{SrTiO}_{3}$ interface explained by microscopic imaging of channel-flow over $\mathrm{SrTiO}_{3}$ domains. ACS Appl. Mater. Inter. 8, 12514-12519 (2016).

47. Lu, H. et al. Mechanical writing of ferroelectric polarization. Science 336, 59-61 (2012)

48. Guo, E. J. et al. Ferroelectric $180^{\circ}$ domain wall motion controlled by biaxial strain. Adv. Mater. 27, 1615-1618 (2015).

49. Cao, Y. et al. Pressure-induced switching in ferroelectrics: phase-field modeling, electrochemistry, flexoelectric effect, and bulk vacancy dynamics. Phys. Rev. B 96, 184109 (2017).

50. Damjanovic, D. A, morphotropic phase boundary system based on polarization rotation and polarization extension. Appl. Phys. Lett. 97, 062906 (2010).

51. Catalan, G. et al. Flexoelectric rotation of polarization in ferroelectric thin films. Nat. Mater. 10, 963-967 (2011).

52. Chen, L. Q. \& Shen, J. Applications of semi-implicit Fourier-spectral method to phase field equations. Comput. Phys. Commun. 108, 147-158 (1998). -. 
53. Haun, M. J. et al. Thermodynamic theory of $\mathrm{PbTiO}_{3}$. J. Appl. Phys. 62, 3331-3338 (1987).

54. Zubko et al. Flexoelectric effect in solids. Annu. Rev. Mater. Res. 43, 387-421 (2013).

\section{Acknowledgements}

The work in Harbin Institute of Technology is supported by the National Natural Science Foundation of China under the contract numbers 11872019 and 51802057, the National Key Research and Development Program of China (2018YFC0705601), and a startup grant from Harbin Institute of Technology, Shenzhen, under project number DD45001017. For work done at UC Berkeley, Z.H.C. acknowledges support from the Army Research Office under grant W911NF-14-1-0104. Y.C. acknowledges the support from the Faculty Science and Technology Acquisition and Retention (STARs) Program in the University of Texas System, the startup funding at the University of Texas at Arlington, and the Texas Advanced Computing Center (TACC) at The University of Texas at Austin for providing HPC resources that have contributed to the research results reported within this paper (URL: http://www.tacc.utexas.edu). R.X. acknowledges support from the National Science Foundation under grant DMR-1708615. S.S. acknowledges support from the U.S. Department of Energy, Office of Science, Office of Basic Energy Sciences, under Award Number DE-SC-0012375 for development of ferroelectric thin films. S.D. acknowledges support from the Gordon and Betty Moore Foundation's EPiQS Initiative, under grant GBMF5307. H.P.W acknowledges the supports from National Science Foundation of China (No. 11672269). J.Y.L. acknowledges support from National Key Research and Development Program of China (2016YFA0201001). L.C. acknowledges the supports from National Natural Science Foundation of China (No. U1532142), Natural Science Foundation of Guangdong Province of China (No. 2015A030310329) and the Science and Technology Research Items of Shenzhen (Grant Nos. JCYJ20160530185705301 and JCYJ20170412153325679). L.W.M. acknowledges support from the U.S. Department of Energy, Office of Science, Office of Basic Energy Sciences, Materials Sciences and Engineering Division under Contract No. DE-AC02-05-CH11231: Materials Project program KC23MP for the development of novel functional materials. The work at Penn State is supported by U.S. National Science Foundation grant under grant number DMR-1744213.

\section{Author contributions}

X.Y.L. and Z.H.C. designed the experiment and analyzed the results with W.W.C. and L.W.M.. Z.H.C. coordinated the project and data analysis. X.Y.L. carried out PFM

experiments and performed the Landau-type phenomenological calculation. Y.C. performed the phase-field simulation in collaboration with L.Q.C., Y.L.T. carried out TEM experiments. S.S. and R.X. grew the films. Z.H.C., Z.Z. and Y.Q.D. carried out the X-ray studies. L.X.Y., Z.H.C. and L.W.M. supervised the study and wrote the manuscript with the help from all other authors.

\section{Additional information}

Supplementary Information accompanies this paper at https://doi.org/10.1038/s41467 019-11825-2.

Competing interests: The authors declare no competing interests.

Reprints and permission information is available online at http://npg.nature.com/ reprintsandpermissions/

Peer Review Information: Nature Communications thanks Hideo Kimura and other anonymous reviewers for their contribution to the peer review of this work.

Publisher's note: Springer Nature remains neutral with regard to jurisdictional claims in published maps and institutional affiliations.

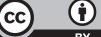

Open Access This article is licensed under a Creative Commons Attribution 4.0 International License, which permits use, sharing, adaptation, distribution and reproduction in any medium or format, as long as you give appropriate credit to the original author(s) and the source, provide a link to the Creative Commons license, and indicate if changes were made. The images or other third party material in this article are included in the article's Creative Commons license, unless indicated otherwise in a credit line to the material. If material is not included in the article's Creative Commons license and your intended use is not permitted by statutory regulation or exceeds the permitted use, you will need to obtain permission directly from the copyright holder. To view a copy of this license, visit http://creativecommons.org/ licenses/by/4.0/

(C) The Author(s) 2019 